\section{In zehn Schritten zur Schmerzkontrolle}

\section{Chronische tägliche Kopfschmerzen plagen zwei bis vier Prozent der Nord- amerikaner und Europäer. Ein 10-Schritte-Plan hilft, die Beschwerden in den Griff zu bekommen.}

$Z_{s}$ chronischen täglichen Kopfschmerzen $(\mathrm{CDH})$ tragen unterschiedliche ätiologische Faktoren bei. Doch trotz aller Unterschiede sollen sich die meisten Schmerzen mithilfe des von Dr. Robert Sheeler von der Mayo Klinik in Rochester vorgestellten 10-SchrittePlans diagnostizieren und behandeln lassen:

1. Ausschließen lebensbedrohlicher oder progressiver sekundärer Kopfschmerzen.

2. Klassifizieren der primären Kopfschmerzen.

3. Identifizieren der verschärfenden und erhaltenden Faktoren.

4. Identifizieren von Komorbiditäten

5. Prüfen der momentanen akuten Therapie.
6. Entgiften bei übermäßigem Medikamentengebrauch.

7. Prüfen vergangener präventiver Therapieversuche.

8. Erstellen eines Kopfschmerzplans mit akuten, präventiven und Lifestyle-Komponenten.

9. Aufklären des Patienten und Anleitung zum Führen eines Kopfschmerztagebuchs.

10. Initiieren eines regelmäßigen Follow-ups zur kontinuierlichen Verbesserung mit dem Ziel tägliche Kopfschmerzen in kontrollierbare episodische Kopfschmerzen zu verwandeln.

Viele Patienten mit chronischen täglichen Kopfschmerzen greifen zu häufig zu Schmerzmitteln, was wiederum „me- dication overuse headaches" (MOH) bedingen kann. Deshalb raten Sheeler und seine Mitarbeiter dazu, die aktuelle Medikation des Patienten zu erfragen und bei übermäßigem Medikamentengebrauch eine Entgiftung zu starten. Zur Unterstützung können Therapeuten über kurze Zeit Kortikosteroide, NSAID oder Dihydroergotamin einsetzen.

Nach Diagnose und Anamnese kann abschließend ein individuell auf den $\mathrm{Pa}$ tienten zugeschnittener Kopfschmerzplan erstellt werden, der präventive sowie akute Therapieoptionen enthält und auch auf die Optimierung des Lebensstils eingeht.

Eine enge Zusammenarbeit zwischen Therapeut und Patient sowie eine regelmäßige Kontrolle des Behandlungserfolgs helfen laut Autoren dabei, langfristig eine gute Kontrolle über $\mathrm{CDH}$ zu erlangen.

Julia Rustemeier

Sheeler RD et al. Chronic Daily Headache: Ten Steps for Primary Care Providers to Regain Control. Headache 2016; online 23. August

\title{
Dem Schmerzsyndrom geht oft posttraumatischer Stress voraus
}

\section{Patienten mit komplexem regionalem Schmerzsyndrom (CRPS) erfüllen häufig auch die Kriterien der posttraumatische Belastungsstörung (PTSD), wie die Ergebnisse einer deutschen Studie zeigen.}

E bromyalgie und andere chronische Schmerzsyndrome gehen mit einer relativ hohen Prävalenz von PTSD einher. Warum sich dies bei CRPS anders verhalten sollte, dafür sah eine Gruppe von Wissenschaftlern der Universitäten Mainz und Erlangen-Nürnberg keine ernsthaften Argumente. Die Mainzer Neurologin Verena Speck und ihre Forscherkollegen machten sich deshalb daran, den Zusammenhang zwischen CRPS und PTSD im Zuge einer Studie $\mathrm{zu}$ untersuchen.

An der Untersuchung waren 152 erwachsene Patienten mit CRPS, davon $71 \%$ Frauen, beteiligt. Weit überwiegend handelte es sich um ein CRPS I, vormals Morbus Sudeck genannt. Rund $11 \%$ litten an CRPS II, früher als Kausalgie be- zeichnet. Von den Schmerzen betroffen waren meist die Hände. 55 Patienten, die ebenfalls an Schmerzen, aber nicht an einem CRPS litten, dienten als Kontrollen, ebenso 55 nach Alter und Geschlecht passende gesunde Probanden. Das Vorliegen von PTSD beurteilten Speck und Mitarbeiter anhand der Resultate einer Befragung mit einschlägigen psychologischen Fragebogen (Posttraumatic Diagnostic Scale, deutsche Version).

Von den CRPS-Patienten wiesen 58 (38\%) eine PTSD auf. Bei den Schmerzpatienten ohne CRPS waren es sechs (10\%), bei den Gesunden zwei (4\%). Der Beginn der PTSD lag bei 50 CRPS-Patienten vor dem Beginn des Schmerzsyndroms, bei 20 (35\%) sogar mehr als fünf
Jahre davor. Unter den erlebten Traumata waren schwere Unfälle, lebensbedrohliche Erkrankungen, häusliche Gewalt, Gewalt durch Fremde, Kriegserlebnisse und Vergewaltigungen durch Familienmitglieder oder Fremde.

Signifikant mit CRPS assoziiert war der Schweregrad der PTSD. Zudem bestand ein Zusammenhang zwischen dem Vorliegen von PTSD und fehlangepassten Bewältigungsmechanismen.

Stutzig macht Speck und Kollegen, dass die PTSD dem CRPS so häufig vorausgeht. „Trotz des Querschnittscharakters unserer Studie und der retrospektiven Einschätzung der PTSD legt dies nahe, dass die PTSD ein Risikofaktor für die Entwicklung eines CRPS sein könnte“, schreiben sie. Eine Längsschnittstudie, mit der sich das untersuchen ließe, halten sie für machbar - aber auch für nötig, um zu klären, ob die Therapie der PTSD die Symptome des CRPS lindern kann.

Dr. Robert Bublak

Speck V et al. Increased prevalence of posttraumatic stress disorder in CRPS. Eur J Pain 2016. Online 21. September 